\title{
Torsades De Pointes Induced by Levofloxacin in an Unknown Long QT Syndrome
}

\author{
Julio Cesar Sauza-Sosa ${ }^{1,2}$
}

${ }^{1}$ Echocardiography Department, National Institute of Cardiology "Ignacio Chávez", Mexico

${ }^{2}$ Cardiology Department, The American British Cowdray Medical Center, Mexico

\section{Clinic Case}

A 43-year-old woman arrived to the Emergency Room because of a syncope that lasted approximately $30 \mathrm{~s}$. She denied previous headache, chest pain or any other cardiovascular symptoms before this episode of sudden loss of consciousness.

In her previous medical history, she denied syncope, neurological or respiratory diagnosed diseases. She only referred the history of breast implants without complications 5 years ago, she denied any other chronic disease. She denied drug allergy history, history of trauma and any other prior hospitalization. Her family history denies sudden death and ischemic heart diseases.

Three days before, she reported flu-like symptoms and she was started on levofloxacin and ibuprofen, she denied the intake of some other concomitant medication. At her arrival, vital signs were stable and physical examination was unremarkable except by the finding of a right periorbital hematoma. Her initial laboratories were CK-MB $3 \mathrm{UI} / \mathrm{L}$, ultrasensitive cardiac troponin I $0.70 \mathrm{pg} / \mathrm{mL}$, TSH $3.26 \mu \mathrm{UI} / \mathrm{mL}$, $\mathrm{Na} 143 \mathrm{nEq} / \mathrm{L}, \mathrm{K} 4.8 \mathrm{mEq} / \mathrm{L}, \mathrm{Cl} 109 \mathrm{mEq} / \mathrm{L}, \mathrm{Ca} 9.6 \mathrm{mg} / \mathrm{dL}, \mathrm{Mg} 2.1 \mathrm{mg} /$ $\mathrm{dL}, \mathrm{P} 3.4 \mathrm{mg} / \mathrm{dL}$, hs-CRP $0.06 \mathrm{mg} / \mathrm{dL}$, Creatinine $0.6 \mathrm{mg} / \mathrm{dL}$. Her initial EKG (Figure 1A) showed prolonged QTc interval (QTc $520 \mathrm{~ms}$ ) and ventricular premature complex with $\mathrm{R} / \mathrm{T}$ phenomenon. A transthoracic Echocardiogram showed preserved left ventricular ejection fraction without regional wall motion abnormalities. At the ER the patient had lipothymy, her second EKG is shown in Figure 1B and shows again a long QTc interval (QTc $500 \mathrm{~ms}$ ) and helical ventricular tachycardia (Torsades de Pointes). The clinical suspicion at that moment was a drug induced long-QT syndrome induced by the current medication (levofloxacin).

She was treated with magnesium sulphate and during her EKG monitoring she had an asymptomatic, hemodinamically stable helical ventricular tachycardia (Torsades de Pointes) that does not required electrical cardioversion (Figure 1C).

QTc returned to normal after five days free of levofloxacin. No more episodes of helical ventricular tachycardia were noted. Her last EKG is shown in Figure 1D. A cardiovascular magnetic resonance was performed and did not show any data suggestive of structural abnormality of the heart or any cardiomyopathy and specifically ruled out myocarditis, arrhythmogenic right ventricular cardiomyopathy, hypertrophic cardiomyopathy and non-compaction ones. Due to the high-risk of cardiac sudden death an implantable cardioverter was placed along with the proper instruction to the patient and her family to avoid any drug that potentially could prolong the QT interval. The patient is asymptomatic and free of syncope and no arrhythmic events have been register by the cardioverter, therefore she had not received any internal electrical therapy at all until today. Her relatives are currently under investigation and genetic analysis is planned for the near future to this family.

\section{Discussion}

In patients documented acquired long-QT syndrome is rarely present Torsades de Pointes. Some factors that predispose to QT prolongation and higher risk for this arrhythmia secondary to drug therapy includes: female sex, low left ventricular ejection fraction, older age, left ventricular hypertrophy, ischemia, bradycardia, electrolyte abnormalities and prolonged repolarization [1,2].

Main types of drugs that prolong the QT-interval include antiarrhythmic drugs (dysopiramide, dofetilide, ibutilide, procainamide, quinidine, sotalol, bepridil, amiodarone), anti-infective agents (azole antifungals, fluoroquinolones, macrolide antibiotics, antiretrovirals), antiemetic drugs (domperidone, droperidol), antihistamines (astemizole, hydroxyzine, terfenadine), antipsychotic agents (chlorpromazine, haloperidol, mesoridazine, thioridazine, pimozide) and analgesic drugs (methadone). Other causes, not directly related to drugs, which can prolong QT-interval are electrolyte abnormalities (hypokalemia, hypocalcemia or hypomagnesemia), bradycardia, ischemia, stroke and structural heart disease [1-3].

The correct measure of QT-interval is in lead II of a 12-leads EKG from the onset of the QRS complex to the point where $T$ wave ends and its adequate correction to heart rate by Bazett's formula makes the diagnosis. Bazett's formula obtains QTc dividing QT-interval by the square root of the RR interval (in ms). QTc is normal if it is less than or equals to $450 \mathrm{~ms}$ in men and less than or equals to $470 \mathrm{~ms}$ in women $[4,5]$.

Fluoroquinolones prolong the QT interval by blocking the cardiac voltage-gated rapid potassium channels $\left(\mathrm{I}_{\mathrm{Kr}}\right)$, this adverse effect can predispose by the interaction with other drugs that may prolong the QT interval and for the dosage of fluoroquinolone. The chemical composition of fluoroquinolones also found as a predisposing factor for the increase in QT interval prolongation. The probability of this adverse drug reaction with levofloxacin is described 1 per million prescriptions. Females are more susceptible than males to QTc prolongation caused by drugs, because of the specific regulation by sex hormones of ion channel expression and function [6].

The management of this adverse reaction includes the identification of any precipitating drug, along with aggressive correction of any metabolic abnormality. Patients with prolonged QT-interval associated with syncope or Torsades de Pointes should be admitted to the Hospital for EKG monitoring during the period of withdraw and washout of the precipitating drug allowing the treatment of concomitant arrhythmias, if indicated. In specific cases, where acute

*Corresponding author: Julio C. Sauza-Sosa, National Institute of Cardiology "Ignacio Chávez", Juan Badiano \#1, Col. Sección XVI, Z.P. 14080, Mexico, Tel: +525555732911; E-mail: sauzamd@hotmail.com

Received July 18, 2016; Accepted July 27, 2016; Published August 01, 2016

Citation: Sauza-Sosa JC (2016) Torsades De Pointes Induced by Levofloxacin in an Unknown Long QT Syndrome. Cardiovasc Pharm Open Access 5: 191 doi:10.4176/2329-6607.1000191

Copyright: (c) 2016 Sauza-Sosa JC. This is an open-access article distributed under the terms of the Creative Commons Attribution License, which permits unrestricted use, distribution, and reproduction in any medium, provided the original author and source are credited. 
A

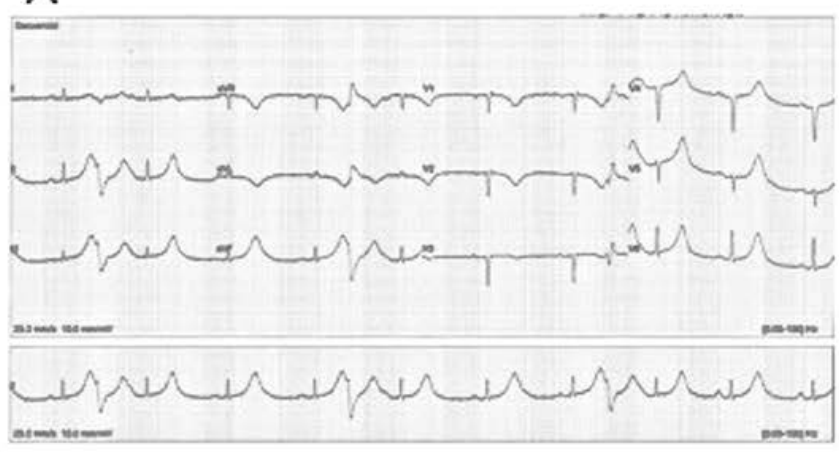

C

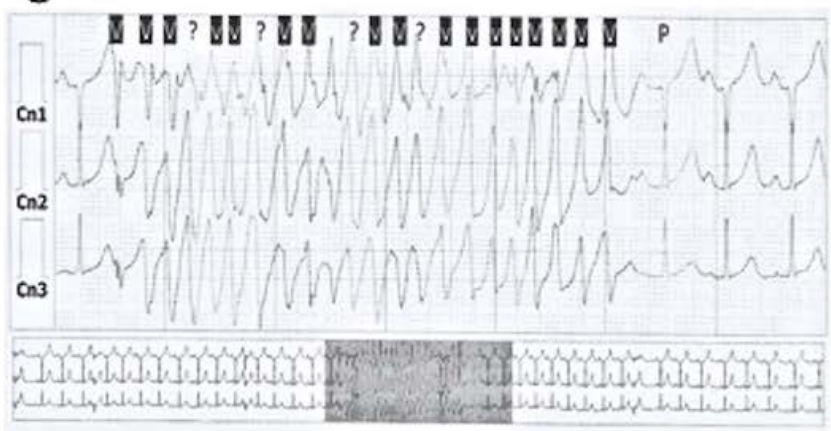

B

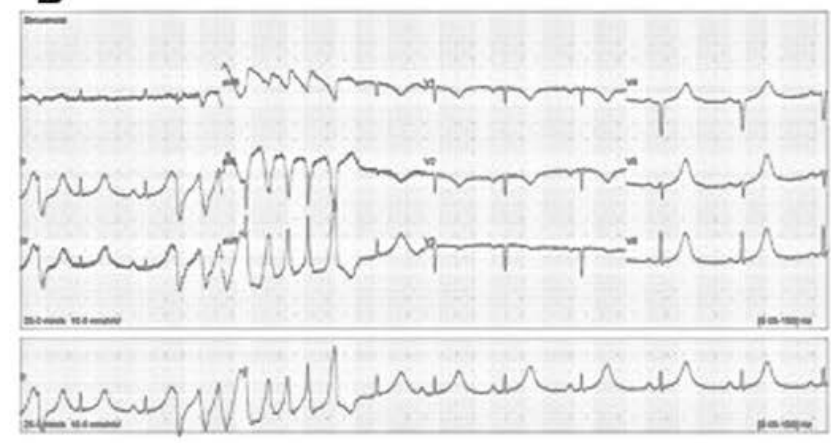

D

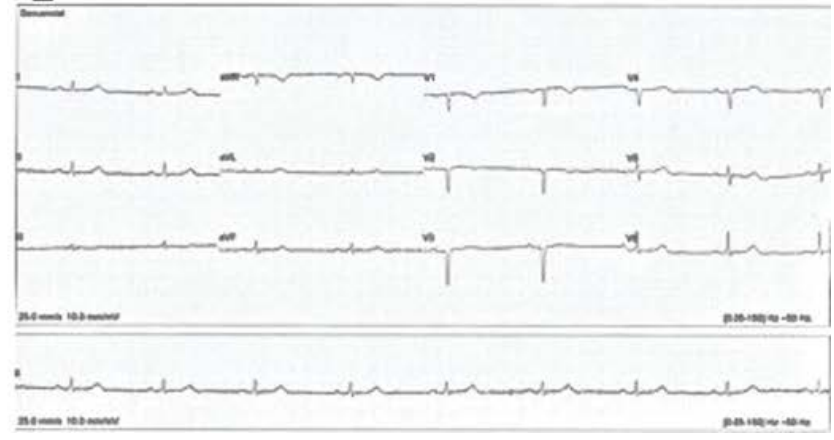

Figure 1: A) EKG that shows long QTc-interval (QTc $530 \mathrm{~ms}$ ) and ventricular premature complex with R/T phenomenon. B) EKG that shows long QTc-interval (QTc 500 $\mathrm{ms}$ ) and helical ventricular tachycardia. C) EKG-Holter monitoring that shows helical ventricular tachycardia. D) EKG that shows a normal QTc (QTc 430 ms) after the resolution of electrocardiographic abnormalities.

therapy is necessary, the proper management includes intravenous magnesium, maintenance of serum potassium levels in the upper limit of normal range, isoproterenol infusion, transient transvenous cardiac pacing, and in some cases the treatment with mexiletine hydrochloride it is also indicated [7].

\section{References}

1. Roden DM (2004) Drug-Induced Prolongation of the QT Interval. N Engl J Med 350: 1013-1022.

2. Passman R, Kadish A (2001) Polymorphic ventricular tachycardia. Long QT syndrome, and torsades de pointes. Med Clin North Am 8: 321.
3. Yap YG, Camm AJ (2003) Drug induced QT prolongation and torsades de pointes. Heart 89: 1363.

4. Al-Khatib SM, Allen NM, Kramer JM, Califf RM (2003) What Clinicians Should About the QT Interval. JAMA 289: 2120-2127.

5. Moss AJ (1993) Measurement of the QT interval and the risk associated with QT interval prolongation: a review. Am J Cardiol 72: 23B.

6. Rubinstein E, Camm J (2002) Cardiotoxicity of fluoroquinolones. J Antimicrob Chemother 49: 593-596.

7. Badri M, Patel A, Patel C, Liu G, Goldstein M, et al. (2015) Mexiletine Prevents Recurrent Torsades de Pointes in Acquired Long QT Syndrome Refractory to Conventional Measures. J Am Coll Cardiol 1: 315-322. 